\title{
The analysis of flow stability in a vortex furnace model
}

\author{
Igor S. Anufriev ${ }^{1}$, Dmitrii O. Glushkov ${ }^{2, *}$, Anatoly P. Papulov ${ }^{1,3}$ and Evgeniy Yu. Shadrin ${ }^{1}$ \\ ${ }^{1}$ Kutateladze Institute of Thermophysics, 630090 Novosibirsk, Russia \\ ${ }^{2}$ National Research Tomsk Polytechnic University, 634050 Tomsk, Russia \\ ${ }^{3}$ Novosibirsk State University, 630090 Novosibirsk, Russia
}

\begin{abstract}
Results of experimental study of the pulsation characteristics of a flow in isothermal model of vortex furnace with vertically oriented nozzles of secondary blast are obtained. With use of laser Doppler measuring system and pressure pulsations analyzer the data about the pressure and velocity pulsations has been received. Spectra of pressure and velocity pulsations at various regime parameters are presented. Absence of non-stationary structures, such as precessing vortex core of a flow, is shown.
\end{abstract}

\section{Introduction}

The targeted wide use of low-grade coal in the energy sector requires creating new efficient and ecologically safe combustion technologies for energy generation. One of the promising technologies in this field is pulverized coal combustion in a vortex flow. The key stage in the development of furnaces using the vortex combustion technology is the detailed study of their internal aerodynamics in the laboratory models. It is known that the high swirling flows under certain conditions are characterized by the loss in stability of the stationary regime, which may be expressed in the appearance of the precessing vortex core (PVC). Intensive pressure pulsations associated with the PVC cause wear of power plants and deterioration of the performance of vortex devices and have a negative impact on the furnace processes. In previous works $[1,2]$ authors visualized the vortex structure of the flow in the model of the improved vortex furnace with distributed fuel-air mixture supply and vertically oriented nozzles of the secondary blast. The aim of this work is to experimentally study the pulsation characteristics.

\section{Experimental setup and measurement techniques}

The scheme of the experimental setup with the mounted three-component (3D-) LDA system is presented at figure 1 . Dimensions of the model are XYZ $320 \times 1200 \times 256 \mathrm{~mm}$, and the diameter of the vortex combustion chamber is $320 \mathrm{~mm}$. A distinctive feature of this

\footnotetext{
${ }^{*}$ Corresponding author: dmitriyog@,tpu.ru
} 
design compared to the previously studied one [3] is the vertical arrangement of the nozzles of the secondary blast, and the presence of the "visor" inside the vortex chamber to prevent entrainment of fuel particles from the combustion chamber.

Measurements of pressure fluctuations were carried out using noise analyzer Bruel\&Kjaer (the limit of pressure measurement up to $103.5 \mathrm{kPa}$, measurement frequency of $4.2 \mathrm{~Hz} \div 20 \mathrm{kHz}$, sensitivity of $54.9 \mathrm{mV} / \mathrm{Pa}$ ). The sensor was placed inside the vortex combustion chamber with a metal sampler - thin-walled tube with a diameter of $2.2 \mathrm{~mm}$ and a length of $160 \mathrm{~mm}$. The transfer function of the sampler is presented in [4]. The measurements were carried out close to the conventional center of the vortex chamber ( $x=y=160 \mathrm{~mm}, z=126 \mathrm{~mm})$ at various operating parameters $(\gamma=1 \div 4$, where $\gamma$ is the ratio of the flow rates through the main and additional nozzles). To diagnose the flow velocity pulsations the two-component (2D-) laser Doppler anemometer LAD-05 (frequency up to 3 $\mathrm{kHz}$ ) was used. The measurements were performed in the range of Reynolds number $3 \cdot 10^{5}<\operatorname{Re}<6 \cdot 10^{5}$, calculated on the diameter of the vortex chamber $(320 \mathrm{~mm})$ and the velocity module in the top burners $\left(V_{0}=5 \div 25 \mathrm{~m} / \mathrm{s}\right)$.

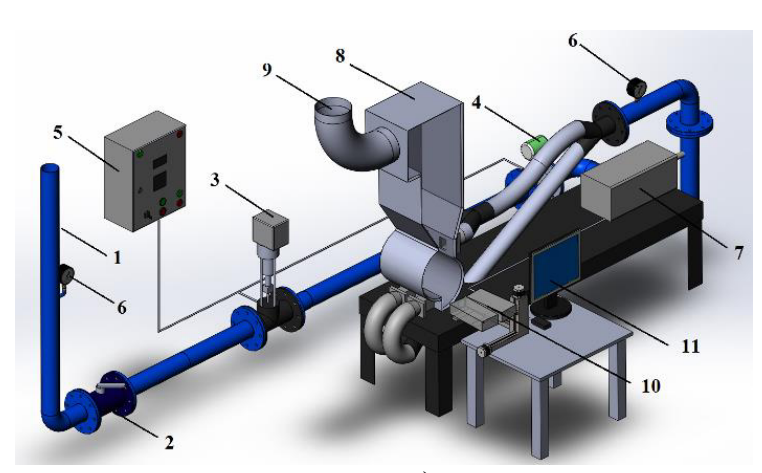

a)

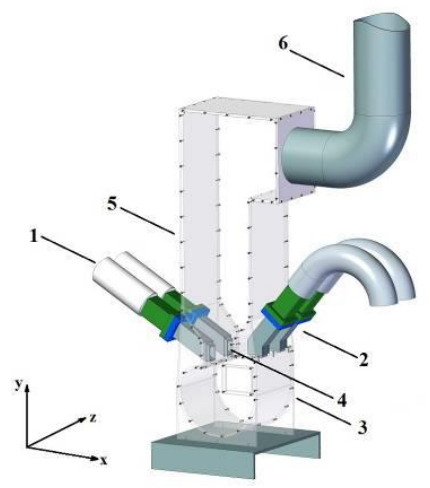

b)

Fig. 1. (a) Scheme of experimental stand: 1 - supply line of compressed air, 2 - stop valve, 3 - shutoff control electrically operated valve, 4 - flow converter, 5 - control cabinet, 6 - manometer, 7 - fog generator, 8 - model of the vortex furnace, 9 - ventilation, 10 -measuring system, 11 - computer. (b) Scheme of a laboratory model of the improved vortex furnace: 1 - main nozzles, 2 - secondary nozzle, 3 - combustion chamber, 4 - diffuser, 5 - cooling chamber, 6 - flue.

\section{Measurement results and their analysis}

Earlier, based on independent measurement techniques, using 3D-LDA [1] and Stereo PIV (Particle Image Velocimetry) systems [2], the structure of the vortex core has been visualized using different criteria [5] and compared with numerical results [6]. To analyze stability of the revealed vortex structure the pressure fluctuations were measured in the central part of combustion chamber. Spectra for the microphone signal obtained for different ratios $\gamma$ are presented in figure 2. They have a complex form with multiple peaks. However, the position of these peaks neither depends on the flow rate ratio, nor on the magnitude of the total flow, which indicates the absence of the unsteady vortex structures (such as PVC [7]) and the presented peaks characterize the model as an acoustic resonator.

The noise analyzer used in the measurements is limited in frequency range. Therefore, the analysis of pulsations at low frequencies $(<5 \mathrm{~Hz})$ was performed with the use of the laser Doppler anemometer LAD-05. The measurements were carried out at different points of the model in the vicinity of the conventional axis of the curved vortex core and near the entrance nozzles, for different values of initial velocity. Figure 3 shows typical spectra of 
pulsations of the horizontal velocity component in the point $(x=150 \mathrm{~mm} ; y=100 \mathrm{~mm}, z=64$ $\mathrm{mm}$ ) in a plane passing through the centers of the nozzles. The complete absence of peaks confirms the earlier conclusion about the PVC absence. These spectra are characteristic for all points of measurements.
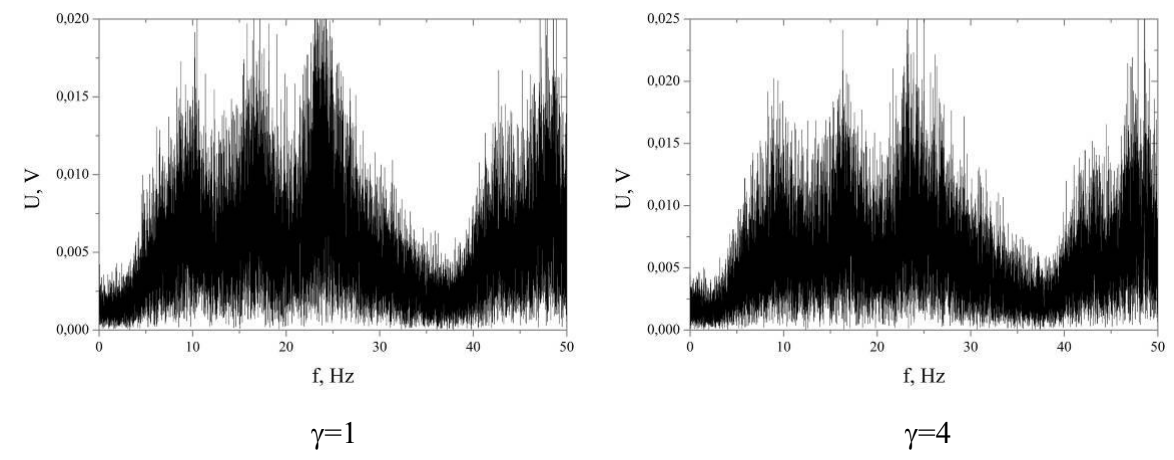

Fig. 2. Spectra of pressure pulsations in the center of the combustion chamber at different ratios $\gamma$.
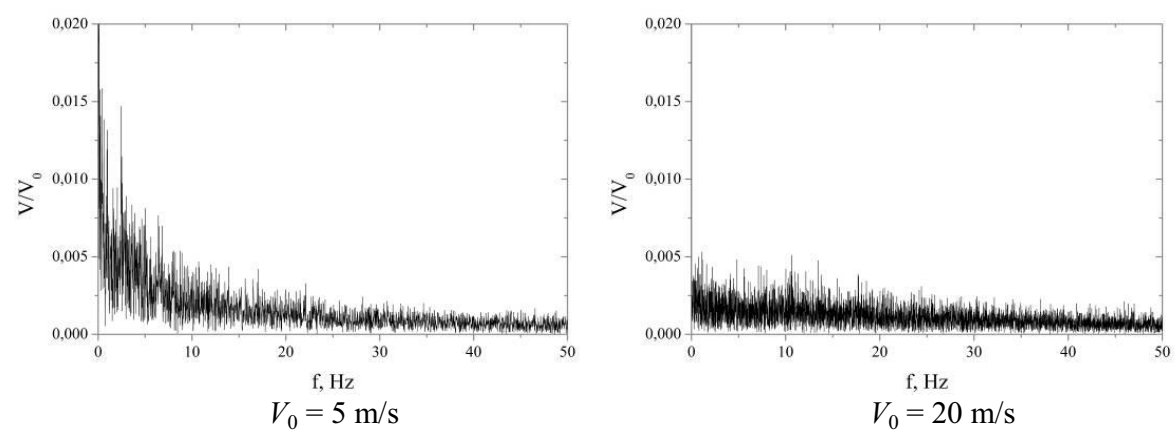

Fig. 3. Spectra of the $x$-velocity component pulsations for different values of initial velocity $V_{0}(\gamma=1)$.

\section{Conclusions}

Using the modern methods of measurements the pulsation characteristics of the swirling flow have been investigated in the improved model of the vortex furnace with distributed fuel-air supply and vertically oriented nozzles of the secondary blast. The results of the spectral analysis of pulsations of pressure and velocity of the turbulent swirling flow with different prove the stability of the stationary structure of the vortex core flow in the studied improved model of the vortex furnace. The absence of negative effects associated with the vortex core precession is one of the important practical advantages of the studied furnace design.

Research was supported by the Russian Science Foundation (Project No. 14-19-00137).

\section{References}

1. I. S. Anufriev, P.A. Strizhak, M.Yu. et al, Tech. Phys. Letters. 41, 727 (2015)

2. I.S. Anufriev, P.A. Kuibin, E.Yu. et al, Thermophys. Aeromech. 6, 621 (2016)

3. D.V. Krasinsky, V.V. Salomatov et al, Therm. Eng. 62, 117 (2015)

4. I.V. Litvinov, S.I. Shtork et al, Int. J. Heat Fluid Fl. 42, 251 (2013)

5. Y. Dubief, F. Delcayre, J. Turbulence. 1, 22 (2000) 
6. D.V. Krasinsky, O.V. Sharypov, J. Eng. Thermophys. 24, 348 (2015)

7. V.L. Okulov, I.V. Naumov, J.N. Sorensen, Tech. Phys. 52, 583 (2007) 